\title{
Super-thin single crystal diamond membrane radiation detectors
}

Cite as: Appl. Phys. Lett. 103, 112106 (2013); https://doi.org/10.1063/1.4821035

Submitted: 19 July 2013 . Accepted: 28 August 2013 . Published Online: 12 September 2013

Michal Pomorski, Benoit Caylar, and Philippe Bergonzo
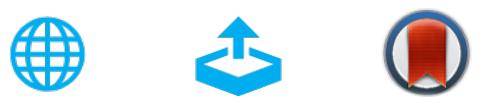

View Online

Export Citation

CrossMark

\section{ARTICLES YOU MAY BE INTERESTED IN}

An ultra-thin diamond membrane as a transmission particle detector and vacuum window for external microbeams

Applied Physics Letters 103, 243106 (2013); https://doi.org/10.1063/1.4833236

Single crystal CVD diamond membranes for betavoltaic cells

Applied Physics Letters 108, 252105 (2016); https://doi.org/10.1063/1.4954013

Charge multiplication effect in thin diamond films

Applied Physics Letters 109, 043502 (2016); https://doi.org/10.1063/1.4959863

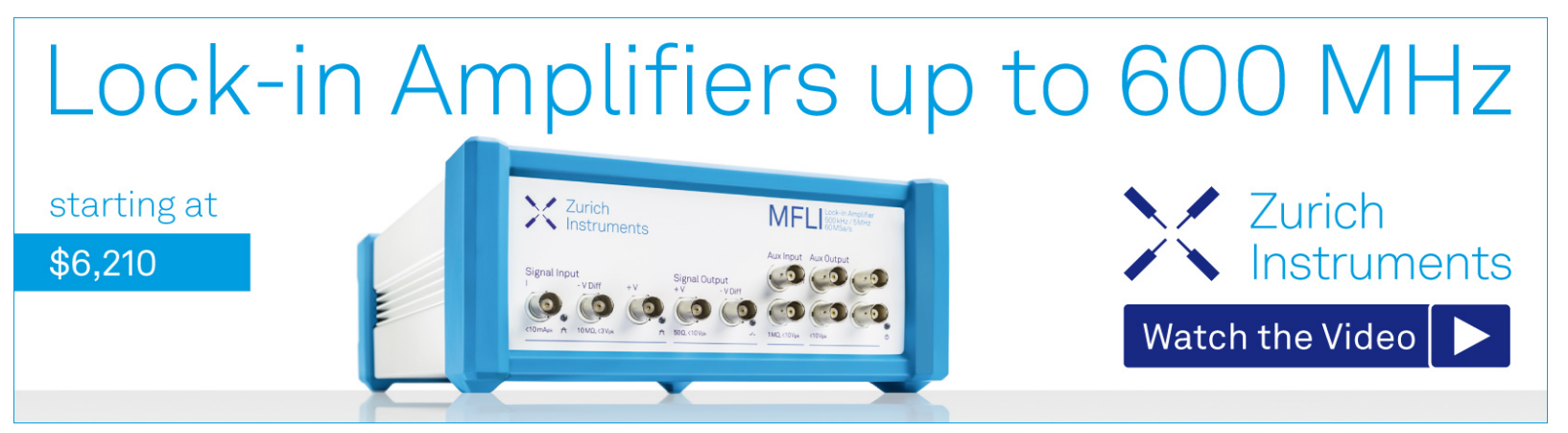




\title{
Super-thin single crystal diamond membrane radiation detectors
}

\author{
Michal Pomorski, Benoit Caylar, and Philippe Bergonzo \\ CEA-LIST, Diamond Sensors Laboratory, Gif-sur-Yvette F-91191, France
}

(Received 19 July 2013; accepted 28 August 2013; published online 12 September 2013)

\begin{abstract}
We propose to use the non-electronic grade (nitrogen content $5 \mathrm{ppb}<[\mathrm{N}]<5 \mathrm{ppm}$ ) single crystal (sc) chemical vapour deposited (CVD) diamond as a thin-membrane radiation detector. Using deep $\mathrm{Ar} / \mathrm{O}_{2}$ plasma etching it is possible to produce self-supported few micrometres thick scCVD membranes of a size approaching $7 \mathrm{~mm} \times 7 \mathrm{~mm}$, with a very good surface quality. After metallization and contacting, electrical properties of diamond membrane detectors were probed with $5.486 \mathrm{MeV} \alpha$-particles as an ionization source. Despite nitrogen impurity, scCVD membrane detectors exhibit stable operation, charge collection efficiency close to $100 \%$, with homogenous response, and extraordinary dielectric strength up to $30 \mathrm{~V} / \mu \mathrm{m}$. (C 2013 AIP Publishing LLC. [http://dx.doi.org/10.1063/1.4821035]
\end{abstract}

Since its first use as a solid state ionization chamber, diamond is known as a perfect material for radiation detectors to be used in harsh environments due to its striking electronic properties, such as high carrier mobility, wide band-gap, radiation hardness, and high breakdown voltage. Although natural single crystal (sc) diamond were used already from 50 s, a real breakthrough in diamond detectors development took place in 2002 when first results on extraordinary long life time and very high mobilities of charge carriers synthetic scCVD electronic grade (scCVD-EG) diamond grown by Element 6 were reported. ${ }^{1}$ Since that date, scCVD-EG diamonds detectors were successfully fabricated to the benefit of several physics experiments, including (i) Large Hadron Collider (LHC) as beam loss monitors, ${ }^{2}$ (ii) GSI (a heavy ions accelerator) as fast start detectors, ${ }^{3,4}$ and (iii) synchrotrons (ESRF, Soleil, Brookhaven, Spring-8) as semitransparent $\mathrm{x}$-ray beam monitors. ${ }^{5-7}$ The scCVD-EG detectors are commercialized today ${ }^{8}$ although in parallel new $R \& D$ activities are ongoing on synthetic diamond detectors aiming at further improvement of scCVD devices such as improving the device dimensions, e.g., from heteroepitaxial approaches $^{9}$ or/and its radiation hardness. For example, recent developments in $3 \mathrm{D}$ diamond detectors ${ }^{10,11}$ rely on the reduction of the charge drift path keeping the standard thickness of the sensors. In the case of minimum ionizing particles or fast neutrons, this allows the creation of a higher number of free-charge carriers and superior (to planar geometry detectors) radiation hardness. However for certain applications, including low energy heavy ions detection, low energy X-ray beam position monitors, thermal neutron detection, or UV detection, the induced signals are rather high intensity, and interactions take place in the first few micrometers length, so thin detection volumes of a thickness tuned to this short distance can be used (or must be used, if detector transparency is a critical issue). One alternative solution for thin detection volumes fabrication using diamond material would be to use heteroepitaxial polycrystalline diamond films (pcCVD) on $\mathrm{Si}$ substrates. The $\mathrm{Si}$ wafer can be easily locally etched off leaving auto-supported pcCVD diamond membranes. However, the use of pcCVD as radiation detection in the past have shown that the user must cope with several serious drawbacks including lack of energy resolution, erratic and persistent currents, ${ }^{12}$ polarization and priming phenomena-all these arising from the presence of sp2 phase in form of grain boundaries. ${ }^{13}$ Especially for super-thin pcCVD films where the sp2/sp3 ratio is high, these effects amplify rendering pcCVD membranes unreliable to use for several of the radiation metrology applications. Natural choice thus has often considered the sole use of scCVD-EG diamond. Nevertheless, when considering typically a $10 \mu \mathrm{m}$ thick membrane and taking the drift velocity vs. E field relation in $\langle 100\rangle$ oriented scCVD diamond, the charge transient time at high $\mathrm{E}>5 \mathrm{~V} / \mu \mathrm{m}$ will be no longer than $100 \mathrm{ps}^{14}$ In such conditions, the use of a non-electronic grade (short life time of the charge carriers due to the presence of atomic impurities) scCVD material could remain potentially attractive. In the following we show a method of fabrication of self-supported diamond membrane detectors based on commercially available cheap, so called 'optical grade' scCVD (scCVD-OG) material (nitrogen content $5 \mathrm{ppb}<[\mathrm{N}]<5 \mathrm{ppm}$ ) of detection characteristics identical to thick scCVD-EG detectors. ${ }^{14}$

Prior to selective plasma etching of membranes, scCVD-OG samples were pre-thinned using two consecutive steps: laser slicing and further mechanical polishing (lapping). Employing these methods plates down to $20 \mu \mathrm{m}$ can be obtained; however, for higher mechanical stability of the self-supported membranes we choose $40 \mu \mathrm{m}$ thick plates for further processing, which are more robust with respect to manual handling. An $\mathrm{Ar} / \mathrm{O}_{2}$ plasma etching method using a magnetron sputtering Physical Vapour Deposition (PVD) system (PLASSYS) was used to locally etch down the prethinned scCVD diamond plates. This PVD apparatus in normal condition is used for thin films sputtering; here, for the purposes of plasma etching the magnetron induced magnetic field (enhancing sputtering yield, but inducing unhomogeneities of the plasma) was blocked using a thick $\mu$-metal (alloy Ni-Fe) layer, and the diamond sample was placed at the sputtering target place. Patterning on the sample regions not to be exposed to the plasma were simply masked with a laser cut pcCVD diamond shadow mask, laid on the top of the scCVD diamond. Following parameters were set during the process; gas flow: $\operatorname{Ar} 32 \mathrm{sscm}, \mathrm{O}_{2}$ $32 \mathrm{sscm}$; pressure $8.4 \mathrm{mbar}$, RF power $200 \mathrm{~W}$. Using such 
(a)

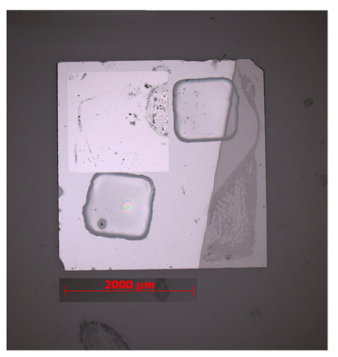

(b)

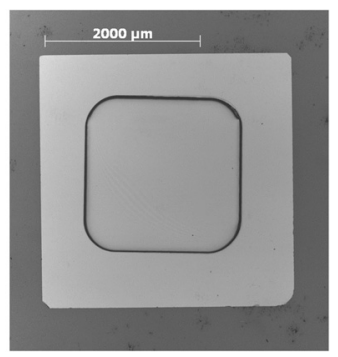

FIG. 1. (a) A $6.5 \mu \mathrm{m}$ thick scCVD diamond membrane used for radiation detector fabrication. (b) Defect-less bare $2 \times 2 \mathrm{~mm}^{2}$ and $3 \mu \mathrm{m}$ thick membrane etched in $40 \mu \mathrm{m}$ thick $3 \times 3 \mathrm{~mm} \mathrm{scCVD}$ diamond.

conditions, the diamond etching rate was in the $1 \mu \mathrm{m} / \mathrm{h}$ range.

Figure 1 displays two scCVD plates etched with $\mathrm{Ar} / \mathrm{O}_{2}$ plasma process: (a) Already Al metalized sample with three small membranes of $1 \times 1 \mathrm{~mm}^{2}$ area (from left upper corner): $6.5 \mu \mathrm{m}$ (Al coated), $10 \mu \mathrm{m}, 1 \mu \mathrm{m}$ thick, respectively; (b) A larger area $2 \times 2 \mathrm{~mm}^{2}$ defect-free membrane of and $3 \mu \mathrm{m}$ thickness etched in $3 \times 3 \mathrm{~mm} 40 \mu \mathrm{m}$ thick sample. Selfsupported membranes of up to $7 \times 7 \mathrm{~mm}^{2}$ and $1 \mu \mathrm{m}$ thick can be fabricated using this method on commercial available scCVD-OG diamond plates. The surface quality of the plasma etched regions was evaluated using AFM (see Figure 2) and optical interferometry technique. We do not observe any significant changes in surface morphology and roughness after etching up to $60 \mu \mathrm{m}$ of diamond, contrary to the results reported for deep RIE etching. ${ }^{15}$

The rms roughness of the surface remains below a few $\mathrm{nm}$, corresponding to the specification of pristine scCVDOG samples. ${ }^{16}$ Using optical interferometry we also probed the macroscopic topography of the etched areas; the surface bow below $150 \mathrm{~nm}$ was measured for several samples over areas up to $3 \times 3 \mathrm{~mm}^{2}$. Although negligible in case of few micrometers thick membranes, it can be unwelcome if membranes below $1 \mu \mathrm{m}$ are required. It is not clear to us at current state of development if the bow comes from the etching process or mechanical polishing. Apart from the surface bow, other limiting factors for super-thin membrane production can be identified: (i) The wedge of the pre-thinned scCVD plates. In average wedges from 0.2 to $6 \mu \mathrm{m}$ over $9 \mathrm{~mm}^{2}$ areas were measured with optical interferometry on several prethinned scCVD-OG plates. (ii) The graphitic inclusions/ voids within the diamond bulk. (iii) The microscopic surface defects arising from mechanical polishing. In Figure 1(a) bottom membrane has thickness of about $1 \mu \mathrm{m}$; a hole in the left-bottom corner can be seen, formed most probably during the plasma etching process due to the presence of an inclusion/void or a large surface defect. These undesirable factors can be avoided by improving the polishing process and by pre-selection of diamond plates.

After $\mathrm{Ar} / \mathrm{O}_{2}$ etching, the membranes were cleaned in a boiling acid solution $\left(\mathrm{H}_{2} \mathrm{SO}_{4}+\mathrm{KNO}_{3}\right)$ to remove possible contamination with graphite. Then, rinsed in Di water and dried in Ar flow. Both surfaces of the $6.5 \mu \mathrm{m}$ thick membrane were coated with about $400 \mathrm{~nm} \mathrm{Al}$ using PVD method (top contact $1.5 \times 1.5 \mathrm{~mm}$, bottom contact full pad, Figure 1(a)). Contacts are patterned using standard contact photolithographic technique. A special care is needed in order not to touch the membrane with tweezers, and here the membrane ring enables its easy handling from its thick frame. About $90 \%$ of processed membranes of 1-10 micrometers thick survived rather harsh treatment (acid boiling, spin coating, ultrasonic cleaning, and multiple handling) that demonstrates the mechanical robustness of the fabricated self-supported structure.

To probe the current-voltage characteristics of metalized $6.5 \mu \mathrm{m}$ thick scCVD-OG diamond membranes, we used a high precision 6517a Keithley electrometer. Measurements are done in vacuum by applying high voltage (from the Keithley voltage source) to the top $1.5 \times 1.5 \mathrm{~mm} \mathrm{Al} \mathrm{elec-}$ trode, while the bottom pad electrode is kept at the input of the pico-ammeter connected to the ground. After applying the voltage step about $5 \mathrm{~min}$ time is needed for the current stabilization meaning: the difference of the last ten current point measurements is less than $10 \%$. If this condition is fulfilled, the final current measurement is taken as an average of the ten single measurements. Results are displayed in Figure 3. Extraordinary dielectric strength is evidenced that at around $200 \mathrm{~V}(30 \mathrm{~V} / \mu \mathrm{m})$ the leakage current remains below $10 \mathrm{nA}$-a level still compatible with solid state detector operation. One can note soft breakdown points at around $100 \mathrm{~V}(15 \mathrm{~V} / \mu \mathrm{m})$ and $-75 \mathrm{~V}(-12 \mathrm{~V} / \mu \mathrm{m})$, where the current starts to increase exponentially. We suspect that most of the leakage current takes place at the vicinity of the top $\mathrm{Al}$ electrode, particularly, at the bottom edge, where some overlap region of the Al electrode with $1 \mu \mathrm{m}$ thick membrane can be seen (Figure 1(a)). In this region $\mathrm{E}$ reaches more than $100 \mathrm{~V} / \mu \mathrm{m}$ at biases $>100 \mathrm{~V}$. This value is commonly reported as the impact ionization threshold (avalanches) in intrinsic scCVD diamond. ${ }^{17}$ Also, at low $\mathrm{E}$ field region -5 to $5 \mathrm{~V} / \mu \mathrm{m}$, an ohmic behavior is observed for $\mathrm{Al}$ contacts on diamond (inset of Figure 2). The CVD diamonds contain structural defects as threading dislocations, which are known to contribute strongly to the dark current in scCVD-EG
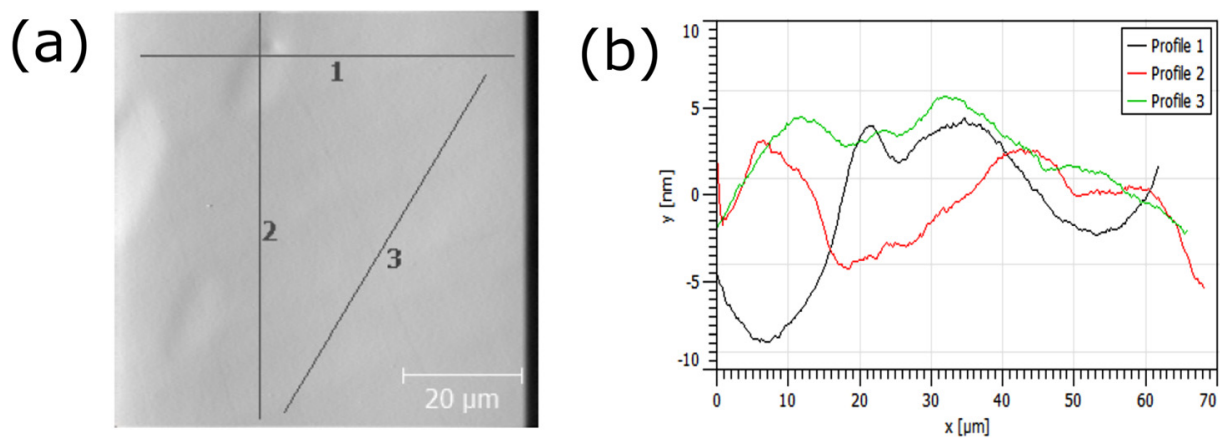

FIG. 2. The AFM topography of a scCVD-OG sample after deep $\mathrm{Ar} / \mathrm{O}_{2}$ plasma etching of $40 \mu \mathrm{m}$. (a) 2D map, (b) corresponding profiles. 


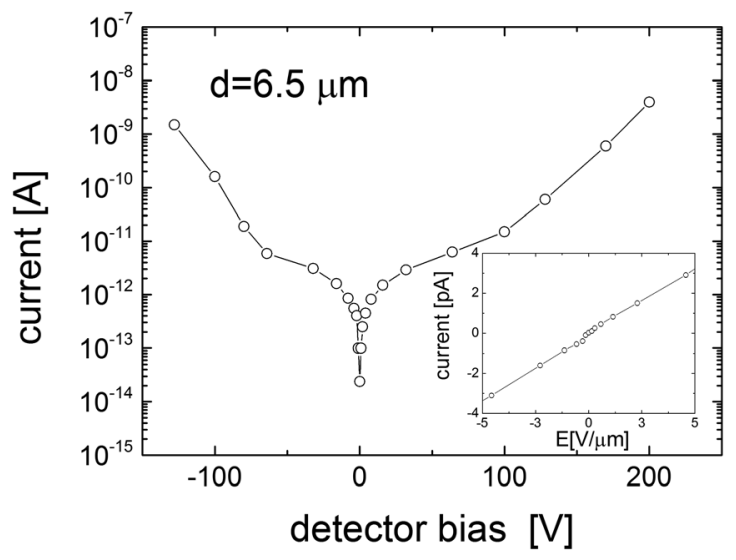

FIG. 3. The I-V characteristics of $6.5 \mu \mathrm{m}$ thick scCVD-OG diamond membrane. (inset) Zoom-in at the low field region, ohmic behavior is evidenced.

diamond $^{18}$ and other large band-gap semiconductor materials. ${ }^{19,20}$ The presence of nitrogen impurity (a deep donor), although significantly decreasing lifetime of the excess charge carriers, seems to be of beneficial influence to the dielectric strength, by passivating the shallow states of dislocations, just as radiation induced defects (also deep donors) in scCVD-EG as reported earlier. ${ }^{21}$

A $4 \mathrm{kBq}^{241} \mathrm{Am} 5.486 \mathrm{MeV} \alpha$-particles spectroscopic grade source was used to probe charge collection efficiency (CCE) of membrane sensor. In order to only characterize the membrane, only the signal induced by alpha particles traversing the membrane should be acquired. Since range of $5.486 \mathrm{MeV} \alpha$-particles is about $12 \mu \mathrm{m}$ in diamond, some particles are stopped within the metalized thick frame depositing full energy, while others will traverse the membrane (thickness $<12 \mu \mathrm{m}$ ) depositing only a part of its energy, thus a selection of $\alpha$-particle's hits is necessary. For this purpose we used a $\Delta \mathrm{E}-\mathrm{E}$ detectors set-up presented in Figure 4 . The scCVD-OG diamond membrane detector $(\Delta \mathrm{E})$ is placed on the top of a thick $(300 \mu \mathrm{m})$ scCVD-EG detector $(\mathrm{E})$, both connected to fast charge sensitive amplifiers. ${ }^{22}$ Amplified signals from both diamond detectors are digitalized and analyzed on a LeCroy $4 \mathrm{GHz}$ digital storage oscilloscope (DSO) using its internal mathematical functions-measurement of signals amplitudes (proportional to the collected charge) in a trend. Only $\alpha$-particles which pass through the membrane can reach a thick scCVD-EG diamond detector placed beneath, where they are stopped inducing pulses used also as a signal trigger for the signal from the membrane detector. This way, both amplitudes of induced signals from $\Delta \mathrm{E}+\mathrm{E}$ detectors are recorded on the DSO in a shot-by-shot base for later off-line analysis.

For thin-absorbers and traversing particles the Gaussian fit is not anymore applicable. Instead, we used the Vavilov distribution (thick black curve in Fig. 5(a)) ${ }^{23}$ to fit the pulseheight spectra of the membrane detector, extracting two parameters: the most probable value (MPV) and $\sigma$ the width of the distribution. Figure 5(a) displays the pulse height spectra of $5.486 \mathrm{MeV} \alpha$-particles measured using the $\Delta \mathrm{E}$-E detectors set-up. The two perfectly overlapped spectra (most to the left) correspond to the signals measured by the $\Delta \mathrm{E}$ detector (a scCVD-OG membrane) at $\pm 100 \mathrm{~V}$ detector polarities $(\mathrm{E} \sim 14 \mathrm{~V} / \mu \mathrm{m})$ peaking at around $\mathrm{MPV}=1.82 \mathrm{MeV}$. The spectrum in the center (peaking at $3.52 \mathrm{MeV}$ ) is the response of the E detector (a thick scCVD-EG) for the $\alpha$-particles traversing the membrane. The energy loss within the membrane of $5.486 \mathrm{MeV} \alpha$-particles corresponds to a diamond thickness
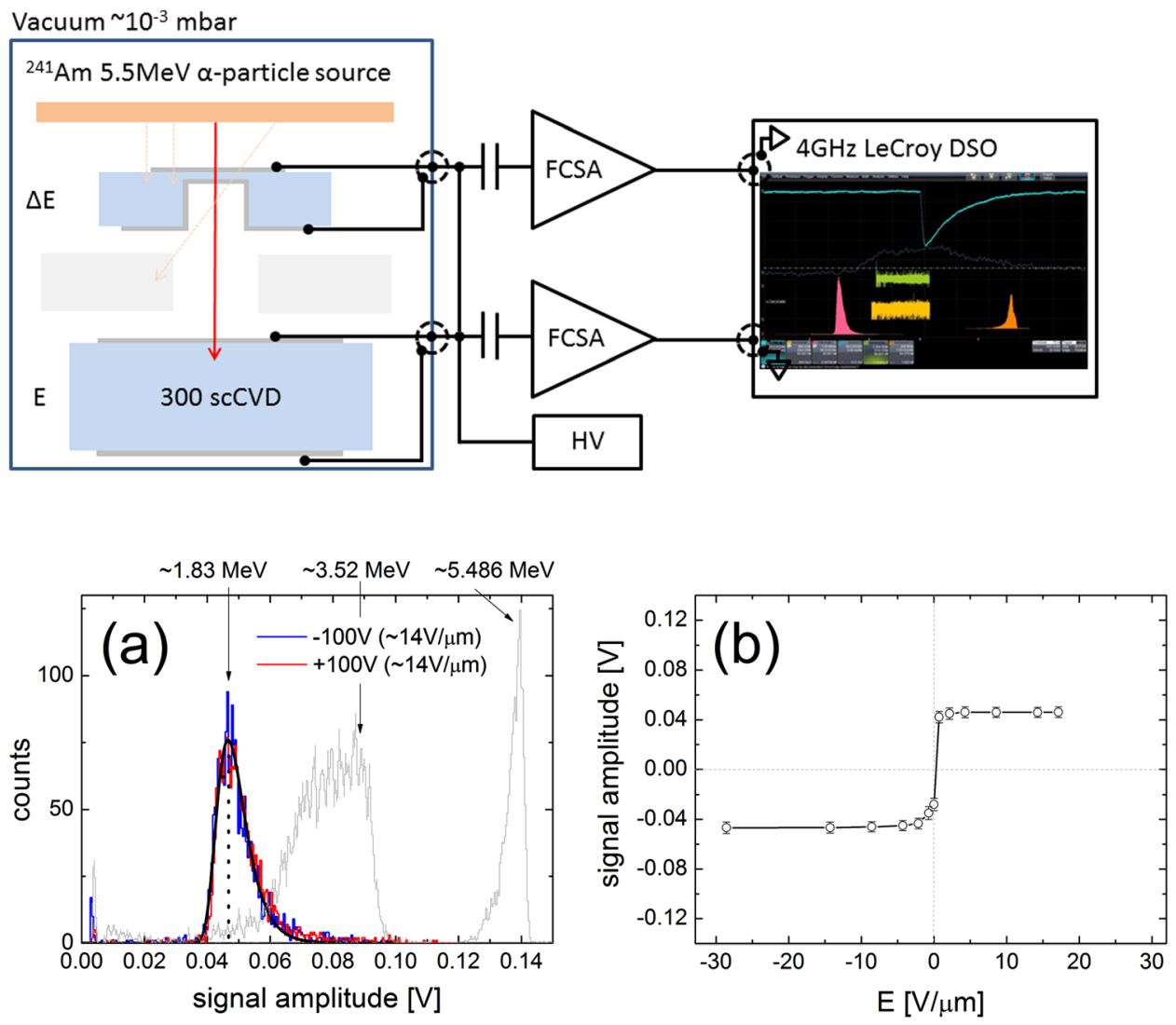

FIG. 4. A $\Delta \mathrm{E}-\mathrm{E}$ set-up used for scCVD-OG membrane charge collection efficiency measurement.
FIG. 5. (a) Spectra of $5.486 \mathrm{MeV}$ alpha-particles: (most to the left) acquired with $\triangle \mathrm{E}$ membrane scCVDOG detector (center) acquired with $\mathrm{E}$ thick scCVD-EG diamond detector while membrane in-the-way (most to the right) acquired with scCVD-EG diamond detector while no membrane in-the-way. (b) The collected charge vs. applied electric field for scCVDOG membrane detector. 
of about $6.5 \mu \mathrm{m}$ according to SRIM calculations, ${ }^{24}$ and this value was confirmed from optical interferometry measurements. The spectrum most to the right is the pulse-height of $\alpha$-particles measured by $\mathrm{E}$ detector while $\Delta \mathrm{E}$ detector was removed, distribution peaking at $5.486 \mathrm{MeV}$ (we assume full CCE for scCVD-EG material) - a full energy of ${ }^{241} \mathrm{Am}$ emitted $\alpha$-particles. The sum of $\Delta \mathrm{E}+\mathrm{E}$ signals gives $5.35 \mathrm{MeV}$ which is lower by about $136 \mathrm{keV}$ than full energy of emitted $\alpha$-particles. The energy loss in $400 \mathrm{~nm} \mathrm{Al}$ entrance electrode was estimated to about $65 \mathrm{keV}$, which leaves us with $71 \mathrm{keV}$, thus corresponding to about $1.3 \%$ (value within measurement uncertainty of $\sigma_{\text {vavilov }}=10 \%$ ) of total $\alpha$-particles energy; thus, we assume CCE close to $100 \%$.

Figure 5(b) displays the MPVs $s_{\text {vavilov }}$ vs. electric filed applied to the membrane detector with $\sigma \mathrm{s}_{\mathrm{vavilov}}$ as the measurement uncertainty. We observe a quick saturation of the characteristics at relatively low E for both polarities, similar to the thick scCVD-EG diamond detectors, again a strong evidence of full CCE. Finally, the $\Delta \mathrm{E}$ detector was left over $24 \mathrm{~h}$ of continuous counting, we did not observe any shift or broadening of peak position or width; thus, the absence of observable priming or polarization phenomena present in thick diamond material containing electrically active defects ${ }^{25}$ was demonstrated.

We presented a method of fabrication of super-thin scCVD-OG diamond membrane detectors using $\mathrm{Ar} / \mathrm{O}$ plasma etching technique. Self-supported membranes as thin as $1 \mu \mathrm{m}$ and $3 \times 3 \mathrm{~mm}^{2}$ area were produced and characterized as radiation detector. Electrical characterization shows extraordinary dielectric strength, full CCE, and stable operation of this type of radiation sensors, regardless of nitrogen contamination. Membrane scCVD-OG detectors are perfect candidates for applications were transparency and/or radiation hardness are required. Several experiments were performed using scCVD-OG membranes detectors including $\mathrm{X}$-ray beam monitors (XBPM) for low energy X-rays at modern light sources, low energy heavy ion detection and vacuum window for cell irradiation studies, and in-core thermal neutron detection of nuclear reactor. In all these measurements scCVD-OG membranes detector exhibited perfect detection characteristics. More publications on mentioned subjects are currently in preparation.

Authors would like to thank John Morse, ESRF for providing us with pre-thinned scCVD-OG diamond plates and optical measurements.
${ }^{1}$ J. Isberg, J. Hammersberg, E. Johansson, T. Wilkstoem, D. J. Twichen, A. J. Whitehead, S. E. Coe, and G. A. Scarsbrook, Science 297, 1670 (2002).

${ }^{2}$ D. Dobos and H. Pernegger, Nucl. Instrum. Methods Phys. Res. A 628(1), 246 (2011).

${ }^{3}$ M. Ciobanu, E. Berdermann, N. Herrmann, K. D. Hildenbrand, M. Kis, W. Koenig, J. Pietraszko, M. Pomorski, M. Rebisz-Pomorska, and A. Schuettauf, IEEE Trans. Nucl. Sci. 58(4), 2073 (2011).

${ }^{4}$ J. Pietraszko, L. Fabbietti, W. Koenig, and M. Weber, Nucl. Instrum. Methods Phys. Res. 1(618), 121 (2010).

${ }^{5}$ K. Desjardins, D. Duran, S. Hustache, M. Pomorski, and W. Shepard, J. Phys.: Conf. Ser. 425, 212004 (2013).

${ }^{6}$ J. Morse, M. Salome, E. Berdermann, M. Pomorski, J. Grant, V. O'Shea, and P. Ilinski, in MRS Proceedings (2007), Vol. 1039.

${ }^{7}$ J. W. Keister, J. Smedley, E. M. Muller, J. Bohon, and A. Heroux, Nucl. Instrum. Methods Phys. Res. A 649, 91-93 (2011).

${ }^{8}$ Micron Semiconductor, Ltd., Marlborough Road, Lancing Business Park, Lancing, Sussex, BN15 8SJ, UK.

${ }^{9}$ N. Vaissiere, S. Saada, M. Bouttemy, A. Etcheberry, P. Bergonzo, and J. C. Arnault, Diamond Relat. Mater. 36, 16-23 (2013).

${ }^{10}$ A. Oh, B. Caylar, M. Pomorski, and T. Wengler, Diamond Relat. Mater. 38, 9-13 (2013).

${ }^{11}$ B. Caylar, M. Pomorski, and P. Bergonzo, "Laser-processed three dimensional graphitic electrodes for diamond radiation detectors," Appl. Phys. Lett. 103, 043504 (2013).

${ }^{12}$ S. Mueller, W. de Boer, M. Schneider, A. Sabellek, M. Schmanau, C. Ruehle, T. Schneider, and R. Hall-Wilton, Phys. Status Solidi A 206(9), 2091 (2009).

${ }^{13}$ C. E. Nebel, A. Waltenspiel, M. Stutzmann, M. Paul, and L. Schaefer, Diamond Relat. Mater. 9, 404 (2000).

${ }^{14}$ M. Pomorski, Ph.D. thesis, University of Frankfurt, 2004.

${ }^{15}$ M. Marinelli, E. Milani, G. Prestopino, C. Verona, G. Verona-Rinati, M. Angelone, M. Pillon, V. Kachkanov, N. Tartoni, M. Benetti, D. Cannata, and F. Di Pietrantonio, J. Synchrotron Radiat. 19, 1015 (2012).

${ }^{16}$ Synthetic CVD Diamond, Element six, Product Ref. No. 145-5000248.

${ }^{17}$ T. Watanabe, T. Teraji, T. Ito, Y. Kamakura, and K. Taniguchi, J. Appl. Phys. 95(9), 4866 (2004).

${ }^{18}$ E. Berdermann, M. Pomorski, W. de Boer, M. Ciobanu, S. Dunst, C. Grah, M. Kis, W. Koenig, W. Lange, W. Lohmann, R. Lovrincic, P. Moritz, J. Morse, S. Mueller, A. Pucci, M. Schreck, S. Rahman, and M. Traeger, Diamond Relat. Mater. 19, 358 (2010).

${ }^{19}$ Z.-Q. Fang, D. C. Reynolds, and D. C. Look, J. Electron. Mater. 29(4), 448 (2000).

${ }^{20}$ F. Zhao, M. M. Islam, B. K. Daas, and T. S. Sudarshan, Mater. Lett. 64, 281 (2010).

${ }^{21}$ M. Pomorski, E. Berdermann, W. de Boer, A. Furgeri, C. Sander, and J. Morse, Diamond Relat. Mater. 16, 1066 (2007).

${ }^{22}$ M. Ciobanu, N. Herrmann, K. D. Hilderbrand, T. I. Kang, M. Kis, and A. Schuettauf, IEEE Nuclear Science Symposium Conference Record N3020, 2025 (2008).

${ }^{23}$ P. V. Vavilov, Zh. Eksp. Teor. Fiz. 32, 920 (1957).

${ }^{24} \mathrm{~J}$. Ziegler, SRIM - The stopping and range of ions in matter, simulation software.

${ }^{25}$ M. Guthoff, K. Afanaciev, A. Dabrowski, W. de Boer, W. Lange, W. Lohmann, and D. Stickland, Nucl. Instrum. Methods Phys. Res. A (in press). 